\title{
SMOOTH STABLE AND UNSTABLE MANIFOLDS FOR STOCHASTIC PARTIAL DIFFERENTIAL EQUATIONS
}

\author{
JINQIAO DUAN, KENING LU, AND BJÖRN SCHMALFUSS \\ Dedicated to Professor Shui-Nee Chow on his $60^{\text {th }}$ birthday
}

\begin{abstract}
J. Dynamics and Diff. Eqns 2004, in press.
Invariant manifolds are fundamental tools for describing and understanding nonlinear dynamics. In this paper, we present a theory of stable and unstable manifolds for infinite dimensional random dynamical systems generated by a class of stochastic partial differential equations. We first show the existence of Lipschitz continuous stable and unstable manifolds by the Lyapunov-Perron's method. Then, we prove the smoothness of these invariant manifolds.
\end{abstract}

\section{Introduction}

This paper, which is a sequel to [10, is devoted to the existence and smoothness of stable and unstable manifolds for a class of stochastic partial differential equations (PDEs).

We consider a nonlinear stochastic evolution equation with a multiplicative white noise:

$$
\frac{d u}{d t}=A u+F(u)+u \dot{W},
$$

where $A$ is a generator of a $C_{0}$-semigroup $e^{A t}$ satisfying an exponential dichotomy condition, $F(\phi)$ is a Lipschitz continuous operator with $F(0)=0$, and $u \dot{W}$ is a noise. The precise conditions on them will given in the next section. Some physical systems or fluid systems with noisy perturbations proportional to the state of the system may be modeled by this equation.

In 10, we proved the existence of Lipschitz continuous unstable manifolds for stochastic partial differential equation (11) by using a random graph transform and a generalized fixed point theorem.

In the present paper, we study the existence and smoothness of stable and unstable manifolds for equation (11). In brief, our main results on the stable and unstable manifolds manifolds may be summarized as follows (the precise statements are given in Sections 3-5). We assume that the semigroup $e^{A t}$ satisfies an exponential

1991 Mathematics Subject Classification. Primary: 60H15; Secondary: 37H05, 37L55, 37L25, $37 \mathrm{D} 10$.

Key words and phrases. Invariant manifolds, cocycles, non-autonomous dynamical systems, stochastic partial differential equations, generalized fixed points.

This work was partially supported by NSF 0209326, NSF0200961, and a travel grant from German DFG Schwerpunktprogramm Interagierende zufällige Systeme von hoher Komplexität. 
condition and the Lipschiz constant of $F$ is dominated by a spectrum gap. Then, there exist global Lipschitz continuous stable and unstable manifolds for stochastic partial differential equation (11). Furthermore, if $F$ is $C^{k}$ and a large spectrum gap condition holds, then these stable and unstable manifolds are $C^{k}$ smooth. The manifolds we study here actually are so-called pseudo-stable and pseudo-unstable manifolds which include the usual stable and unstable manifolds. As for the deterministic systems, we do not need the large spectrum condition for the smoothness of the usual stable and unstable manifolds of stochastic partial differential equation (11). When $F$ is a $C^{1}$ function, the conditions for existence of the $C^{1}$ stable and unstable manifolds are the same as those for the existence of Lipschitz manifolds.

In this paper, we also consider a nonlinear stochastic evolution equation with a additive white noise:

$$
\frac{d u}{d t}=A u+F(u)+\dot{W} .
$$

The precise conditions on them will given in the next section. We will see that after random transformations, equation (2) can be regarded as a special case of equation (1).

The approach we use here is based on the Lyapunov-Perron's method. This approach differs from the Hadamard's graph transform method that we used in [10. However, the optimal conditions for the existence of Lipschitz unstable manifolds obtained by these two different methods are the same.

Stable and unstable manifolds play an important role in the study of nonlinear dynamical systems. Hadamard [1] constructed the unstable manifold of a hyperbolic fixed point of a diffeomorphism of the plane using a geometric method. This geometric method is now called Hadamard's graph transform. Lyapunov [13] and Perron [16] constructed the unstable manifold for an equilbrium point by formulating the problem in terms of an integral equation. This method is analytic rather than geometric and now is called the method of Lyapunov and Perron. There is an extensive literature on stable and unstable manifolds for both finite and infinite dimensional deterministic dynamical systems; see Henry [12], Babin and Vishik [2] or Bates et. al. [3] and the references therein.

Recently, there are some works on invariant manifolds for stochastic ordinary differential equations by Wanner [22, Arnold [1, Mohammed and Scheutzow [15], and Schmalfuß [19]. Wanner's method is based on the Banach fixed point theorem on a space composed of functions with particular exponential growth rates. This method is essentially the Liapunov-Perron approach. In contrast to this method, Mohammed and Scheutzow have applied a classical technique due to Ruelle [17 to the stochastic ordinary differential equations driven by semimartingals. Caraballo et. al. 21 have considered invariant manifolds for a stochastic reaction diffusion equation.

In Section 2, we recall some basic concepts and results for random dynamical systems and stochastic partial differential equations (PDEs). We then prove the existence of the Lipschitz stable manifold for the stochastic PDE (11) in Section 3. 
In Section 4, we prove the smoothness of the stable manifold. The results on the unstable manifold for (11) are given in Section 5 .

\section{Stochastic PDEs and Random Dynamical Systems}

In this section, we introduce some basic notations, assumptions, concepts, and results on stochastic partial differential equations and random dynamical systems.

2.1. Stochastic PDEs with a Multiplicative Noise. Let $H$ be an infinite dimensional separable Hilbert space with norm $|\cdot|$. Consider the nonlinear stochastic partial differential equation

$$
\frac{d u}{d t}=A u+F(u)+u \dot{W}
$$

where $u \in H, W(t)$ is the standard $\mathbb{R}$-valued Wiener process on a probability space $(\Omega, \mathcal{F}, \mathbb{P})$, and the generalized time-derivative $\dot{W}$ formally describes a whitein-time noise. Note that $u \dot{W}$ is interpreted as a Stratonovich stochastic differential.

We assume that the linear operator $A: D(A) \rightarrow H$ generates a strongly continuous semigroup $e^{A t}$ on $H$, which satisfies the exponential dichotomy with exponents $\alpha>\beta$ and bound $K$, i.e., there exists a continuous projection $P^{+}$on $H$ such that

(i) $P^{+} e^{A t}=e^{A t} P^{+}$;

(ii) the restriction $\left.e^{A t}\right|_{R\left(P^{+}\right)}, t \geq 0$, is an isomorphism of the range $R\left(P^{+}\right)$of $P^{+}$onto itself, and we define $e^{A t}$ for $t<0$ as the inverse map.

$$
\begin{aligned}
& \left|e^{A t} P^{+} x\right| \leq K e^{\alpha t}|x|, \quad t \leq 0, \\
& \left|e^{A t} P^{-} x\right| \leq K e^{\beta t}|x|, \quad t \geq 0,
\end{aligned}
$$

where $P^{-}=I-P^{+}$. Denote $H^{-}=P^{-} H$ and $H^{+}=P^{+} H$. Then, $H=H^{+} \oplus$ $H^{-}$. We will call $H^{-}$and $H^{+}$the stable subspace and the unstable subspace, respectively.

The nonlinear term $F$ satisfies $F(0)=0$ and is assumed to be Lipschitz continuous on $H$

$$
\left\|P^{ \pm}\left(F\left(x_{1}\right)-F\left(x_{2}\right)\right)\right\|_{H} \leq \operatorname{Lip} F\left\|x_{1}-x_{2}\right\|_{H}
$$

with the Lipschitz constant $\operatorname{Lip} F>0$.

The existence theory for stochastic evolution equations is usually formulated for Ito equations as in Da Prato and Zabczyk [8], Chapter 7. The equivalent Ito equation for (31) is given by

$$
d u=A u d t+F(u) d t+\frac{u}{2} d t+u d W .
$$

Then, for any initial data $x \in H$, there exists a unique solution of (5). For details about the properties of this solution see Da Prato and Zabczyk [8], Chapter 7.

The solution of (5) can be written as a mild solution:

$$
u(t)=e^{A t} x+\int_{0}^{t}\left(e^{A(t-s)}\left(F(u(s))+\frac{u(s)}{2}\right) d s+\int_{0}^{t} e^{A(t-s)} u(s) d W, \quad x \in H\right.
$$


almost surely for any $x \in H$. Note that the theory in [8] requires that the associated probability space $(\Omega, \mathcal{F}, \mathbb{P})$ is complete.

2.2. Random Dynamical Systems. Let us first look at flows on the probability space $(\Omega, \mathcal{F}, \mathbb{P})$. A flow $\theta$ of mappings $\left\{\theta_{t}\right\}_{t \in \mathbb{R}}$ is defined on the sample space $\Omega$ such that

$$
\theta: \mathbb{R} \times \Omega \rightarrow \Omega, \quad \theta_{0}=\mathrm{id}_{\Omega}, \quad \theta_{t_{1}} \circ \theta_{t_{2}}=\theta_{t_{1}+t_{2}}
$$

for $t_{1}, t_{2} \in \mathbb{R}$. This flow is supposed to be $(\mathcal{B}(\mathbb{R}) \otimes \mathcal{F}, \mathcal{F})$-measurable, where $\mathcal{B}(\mathbb{R})$ is the $\sigma$-algebra of Borel sets on the real line $\mathbb{R}$. To have this measurability, it is not allowed to replace $\mathcal{F}$ by its $\mathbb{P}$-completion $\mathcal{F}^{\mathbb{P}}$; see Arnold [1 Page 547. In addition, the measure $\mathbb{P}$ is assumed to be ergodic with respect to $\left\{\theta_{t}\right\}_{t \in \mathbb{R}}$. Then $\theta:=(\Omega, \mathcal{F}, \mathbb{P}, \mathbb{R}, \theta)$ is called a metric dynamical system.

For the SPDE's with a multiplicative noise, we will consider a special but very important metric dynamical system induced by the Wiener process. Let $W(t)$ be a two-sided Wiener process with trajectories in the space $C_{0}(\mathbb{R}, \mathbb{R})$ of real continuous functions defined on $\mathbb{R}$, taking zero value at $t=0$. This set is equipped with the compact open topology. On this set we consider the measurable flow $\theta=\left\{\theta_{t}\right\}_{t \in \mathbb{R}}$, defined by $\theta_{t} \omega=\omega(\cdot+t)-\omega(t)$. The distribution of this process is a measure on $\mathcal{B}\left(C_{0}(\mathbb{R}, \mathbb{R})\right)$ which is called the Wiener measure. Note that this measure is ergodic with respect to the above flow; see the Appendix in Arnold [1]. Later on we will consider, instead of the whole $C_{0}(\mathbb{R}, \mathbb{R})$, a $\left\{\theta_{t}\right\}_{t \in \mathbb{R}}$-invariant subset $\Omega \subset C_{0}(\mathbb{R}, \mathbb{R})$ of $\mathbb{P}$-measure one and the trace $\sigma$-algebra $\mathcal{F}$ of $\mathcal{B}\left(C_{0}(\mathbb{R}, \mathbb{R})\right)$ with

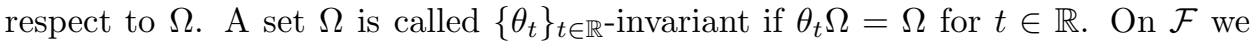
consider the restriction of the Wiener measure also denoted by $\mathbb{P}$.

The dynamics of the system on the state space $H$ over the driven flow $\theta$ is described by a cocycle. For our applications it is sufficient to assume that $\left(H, d_{H}\right)$ is a complete metric space. A cocycle $\phi$ is a mapping:

$$
\phi: \mathbb{R}^{+} \times \Omega \times H \rightarrow H
$$

which is $(\mathcal{B}(\mathbb{R}) \otimes \mathcal{F} \otimes \mathcal{B}(H), \mathcal{F})$-measurable such that

$$
\begin{aligned}
& \phi(0, \omega, x)=x \in H, \\
& \phi\left(t_{1}+t_{2}, \omega, x\right)=\phi\left(t_{2}, \theta_{t_{1}} \omega, \phi\left(t_{1}, \omega, x\right)\right),
\end{aligned}
$$

for $t_{1}, t_{2} \in \mathbb{R}^{+}, \omega \in \Omega$, and $x \in H$. Then $\phi$ together with the metric dynamical system $\theta$ forms a random dynamical system.

2.3. Conjugated Random PDEs. In 10, we used a coordinate transform to convert conjugately a stochastic partial differential equation into an infinite dimensional random dynamical system. Although it is well-known that a large class of partial differential equations with stationary random coefficients as well as Ito stochastic ordinary differential equations generate random dynamical systems (for details see Arnold [1, Chapter 1), this problem is still unsolved for stochastic partial differential equations with a general noise term $C(u) d W$. The reasons are: (i) The stochastic integral is only defined almost surely where the exceptional set may depend on the initial state $x$; (ii) Kolmogorov's theorem, as cited in Kunita 14] Theorem 1.4.1, is only true for finite dimensional random fields; and (iii) the 
cocycle has to be defined for any $\omega \in \Omega$. Nevertheless, for the noise term $u d W$ considered here, we can show that the stochastic PDE (5) indeed generates a random dynamical system.

We considered a linear stochastic differential equation:

$$
d z+z d t=d W .
$$

A solution of this equation is called an Ornstein-Uhlenbeck process. We have the following result.

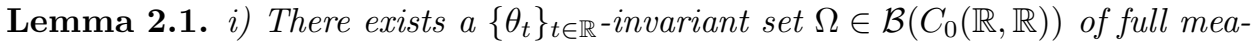
sure with sublinear growth:

$$
\lim _{t \rightarrow \pm \infty} \frac{|\omega(t)|}{|t|}=0, \quad \omega \in \Omega
$$

of $\mathbb{P}$-measure one.

ii) For $\omega \in \Omega$ the random variable

$$
z(\omega)=-\int_{-\infty}^{0} e^{\tau} \omega(\tau) d \tau
$$

exists and generates a unique stationary solution of (7) given by

$$
\Omega \times \mathbb{R} \ni(\omega, t) \rightarrow z\left(\theta_{t} \omega\right)=-\int_{-\infty}^{0} e^{\tau} \theta_{t} \omega(\tau) d \tau=-\int_{-\infty}^{0} e^{\tau} \omega(\tau+t) d \tau+\omega(t) .
$$

The mapping $t \rightarrow z\left(\theta_{t} \omega\right)$ is continuous.

iii) In particular,

$$
\lim _{t \rightarrow \pm \infty} \frac{\left|z\left(\theta_{t} \omega\right)\right|}{|t|}=0 \quad \text { for } \omega \in \Omega
$$

iv) In addition,

$$
\lim _{t \rightarrow \pm \infty} \frac{1}{t} \int_{0}^{t} z\left(\theta_{\tau} \omega\right) d \tau=0 \quad \text { for } \omega \in \Omega .
$$

We now replace $\mathcal{B}\left(C_{0}(\mathbb{R}, \mathbb{R})\right)$ by

$$
\mathcal{F}=\left\{\Omega \cap F, F \in \mathcal{B}\left(C_{0}(\mathbb{R}, \mathbb{R})\right)\right\}
$$

for $\Omega$ given in Lemma 2.1] The probability measure is the restriction of the Wiener measure to this new $\sigma$-algebra, which is also denoted by $\mathbb{P}$. In the following we will consider the metric dynamical system

$$
(\Omega, \mathcal{F}, \mathbb{P}, \mathbb{R}, \theta) .
$$

We show that the solution of (5) defines a random dynamical systems. To see this we consider the following partial differential equation with random coefficients

$$
\frac{d u}{d t}=A u+z\left(\theta_{t} \omega\right) u+G\left(\theta_{t} \omega, u\right), \quad u(0)=x \in H
$$

where $G(\omega, u):=e^{z(\omega)} F\left(e^{-z(\omega)} u\right)$. It is easy ro see that for any $\omega \in \Omega$ the function $G$ has the same global Lipschitz constant $L$ as $F$. In contrast to the stochastic PDE (5), no stochastic differential appears in the random PDE (8). The solution can be interpreted in a mild sense

$$
u(t)=e^{A t+\int_{0}^{t} z\left(\theta_{\tau} \omega\right) d \tau} x+\int_{0}^{t} e^{A(t-s)+\int_{s}^{t} z\left(\theta_{r} \omega\right) d r} G\left(\theta_{s} \omega, u(s)\right) d s .
$$


We note that this equation has a unique solution for each $\omega \in \Omega$. No exceptional sets appear. Hence the solution mapping

$$
(t, \omega, x) \rightarrow u(t, \omega, x)
$$

generates a random dynamical system. Indeed, the mapping $u$ is $(\mathcal{B}(\mathbb{R}) \otimes \mathcal{F} \otimes$ $\mathcal{B}(H), \mathcal{F})$-measurable.

We now introduce the transform

$$
T(\omega, x)=x e^{-z(\omega)}
$$

and its inverse transform

$$
T^{-1}(\omega, x)=x e^{z(\omega)}
$$

for $x \in H$ and $\omega \in \Omega$.

Lemma 2.2. Suppose that $u$ is the random dynamical system generated by (8) Then

$$
(t, \omega, x) \rightarrow T^{-1}\left(\theta_{t} \omega, u(t, \omega, T(\omega, x))\right)=: \hat{u}(t, \omega, x)
$$

is a random dynamical system. For any $x \in H$ this process $(t, \omega) \rightarrow \hat{u}(t, \omega, x)$ is a solution to (1).

Similar transformations were used in Caraballo, Langa and Robinson [21], and Schmalfuß 18.

2.4. Stochastic PDEs with a Additive white noise. We mention another application. We consider a stochastic evolution equation with an additive white noise

$$
\frac{d \hat{u}}{d t}=A \hat{u}+F(\hat{u})+\dot{W}, \quad \hat{u}(0)=x
$$

where $\dot{W}$ is a white noise given as the generalized temporal derivative of a Wiener process with continuous paths in $H$. For simplicity we suppose that $W$ has a covariance with finite trace. For a comprehensive presentation of these equations see [8]. For this problem we have to choose a similar metric dynamical system as above but $\Omega$ is contained in the space of trajectories $C_{0}(\mathbb{R}, H)$.

Suppose that $u^{*}$ is a stationary solution to (13). This means that for the random

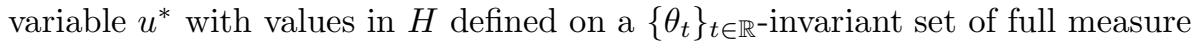

$$
t \rightarrow u^{*}\left(\theta_{t} \omega\right)
$$

is a solution version for (13). It will not be the topic of this article to deal with stationary solutions. For the existence of stable stationary solutions see Caraballo et al. [5].

We now define the nonlinear operator

$$
G(\omega, x)=F\left(x+u^{*}(\omega)\right)-F\left(u^{*}(\omega)\right) .
$$

Note that $G$ has the same Lipschitz constant as $F$. In addition, $G(\omega, 0)=0$. Hence, the problem

$$
\frac{d u}{d t}=A u+G\left(\theta_{t} \omega, u\right), \quad u(0)=x \in H
$$


has a stationary solution which is identical zero. We introduce the random transformations

$$
T(\omega, x)=x-u^{*}(\omega), \quad T^{-1}(\omega, x)=x+u^{*}(\omega) .
$$

Lemma 2.3. Suppose that $u$ is the random dynamical system generated by (14). Then

$$
T^{-1}\left(\theta_{t} \omega, u(t, \omega, T(\omega, x))\right)=: \hat{u}(t, \omega, x)
$$

is a random dynamical system. For any $x \in H$ the process

$$
(t, \omega) \rightarrow \hat{u}(t, \omega, x)
$$

is a solution for (13).

We notice that equation (14) can be regarded as equation (8) with $z=0$. We refer to 4] for more general cases. For the remainder of this article, we consider only equation (8).

2.5. Definition of Invariant Manifolds. We first recall that a multifunction $M=\{M(\omega)\}_{\omega \in \Omega}$ of nonempty closed sets $M(\omega), \omega \in \Omega$, contained in a complete separable metric space $\left(H, d_{H}\right)$ is called a random set if

$$
\omega \rightarrow \inf _{y \in M(\omega)} d_{H}(x, y)
$$

is a random variable for any $x \in H$.

Definition 2.4. A random set $M(\omega)$ is called an invariant set for a random $d y$ namical system $\phi(t, \omega, x)$ if we have

$$
\phi(t, \omega, M(\omega)) \subset M\left(\theta_{t} \omega\right) \text { for } t \geq 0 .
$$

If we can represent $M$ by a graph of a $C^{k}$ (or Lipschitz) mapping

$$
h^{s}(\cdot, \omega): H^{-} \rightarrow H^{+}
$$

such that

$$
M(\omega)=M^{s}(\omega)=\left\{\xi+h^{s}(\xi, \omega) \mid \xi \in H^{-}\right\}
$$

then $M^{s}(\omega)$ is called a $C^{k}$ (or Lipschitz) stable manifold, where $H^{-}$is the stable subspace and $H^{+}$is the unstable subspace, which are introduced in Section 2.1. If we can represent $M$ by a graph of a $C^{k}$ (or Lipschitz) mapping

$$
h^{u}(\cdot, \omega): H^{+} \rightarrow H^{-}
$$

such that

$$
M(\omega)=M^{u}(\omega)=\left\{\xi+h^{u}(\xi, \omega) \mid \xi \in H^{+}\right\}
$$

then $M^{u}(\omega)$ is called a $C^{k}$ (or Lipschitz) unstable manifold. 


\section{Lipschitz Stable Manifolds}

In this section, we first show the existence of a Lipschitz continuous stable manifold for the random partial differential equation

$$
\frac{d u}{d t}=A u+z\left(\theta_{t} \omega\right) u+G\left(\theta_{t} \omega, u\right), \quad u(0)=u_{0} \in H
$$

Then, we apply the inverse transformation $T^{-1}$ to get a stable manifold for the stochastic partial differential equation (15).

Denote by $u\left(t, \omega, u_{0}\right)$ the solution of (15) with the initial data $u\left(0, \omega, u_{0}\right)=u_{0}$. We define the Banach Space for each $\eta, \beta<\eta<\alpha$

$$
C_{\eta}^{+}=\left\{\phi:[0, \infty) \rightarrow H \mid \phi \text { is continuous and } \sup _{t \in[0, \infty)} e^{-\eta t-\int_{0}^{t} z\left(\theta_{\tau} \omega\right) d \tau}|\phi(t)|<\infty\right\}
$$

with the norm

$$
|\phi|_{C_{\eta}^{+}}=\sup _{t \in[0, \infty)} e^{-\eta t-\int_{0}^{t} z\left(\theta_{\tau} \omega\right) d \tau}|\phi(t)| .
$$

Let

$$
M^{s}(\omega)=\left\{u_{0} \in H \mid u\left(\cdot, u_{0}, \omega\right) \in C_{\eta}^{+}\right\}
$$

This is the set of all initial datum through which solutions decay as $e^{\eta t+\int_{0}^{t} z\left(\theta_{\tau} \omega\right) d \tau}$. We shall prove that $M^{s}(\omega)$ is invariant and is given by the graph of a Lipschitz function.

Theorem 3.1. If

$$
K \operatorname{Lip}_{u} G\left(\frac{1}{\eta-\beta}+\frac{1}{\alpha-\eta}\right)<1,
$$

then there exists a Lipschitz invariant stable manifold for the random partial differential equation (15) which is given by

$$
M^{s}(\omega)=\left\{\xi+h^{s}(\xi) \mid \xi \in H^{-}\right\}
$$

where $h^{s}: H^{-} \rightarrow H^{+}$is a Lipschitz continuous mapping and satisfies $h^{s}(0)=$ 0 . Note that $k, \alpha, \beta$ are from the exponential dichotomy condition (4) and $\operatorname{Lip}_{u} G$ denotes the Lipschitz constant of $G(\cdot, u)$ with respect to $u$.

Remark: $\eta=(\alpha+\beta) / 2$ minimizes the quantity

$$
K \operatorname{Lip}_{u} G\left(\frac{1}{\eta-\beta}+\frac{1}{\alpha-\eta}\right)
$$

Proof. We will show that $M^{s}(\omega)$ is given by the graph of a Lipschitz function over $H^{-}$. First we claim that $u^{0} \in M^{s}(\omega)$ if and only if there exists a function $u(\cdot) \in C_{\eta}^{+}$ with $u(0)=u^{0}$ and satisfies

$$
\begin{aligned}
u(t)= & e^{A t+\int_{0}^{t} z\left(\theta_{s} \omega\right) d s} \xi+\int_{0}^{t} e^{A(t-s)+\int_{s}^{t} z\left(\theta_{r} \omega\right) d r} P^{-} G\left(\theta_{s} \omega, u(s)\right) d s \\
& +\int_{\infty}^{t} e^{A(t-s)+\int_{s}^{t} z\left(\theta_{r} \omega\right) d r} P^{+} G\left(\theta_{s} \omega, u(s)\right) d s .
\end{aligned}
$$

where $\xi=P^{-} u^{0}$. 
To prove this claim, first we let $u^{0} \in M^{s}(\omega)$. By using the variation of constants formula, we have that

$$
\begin{aligned}
P^{-} u\left(t, u^{0}, \omega\right)= & e^{A t+\int_{0}^{t} z\left(\theta_{s} \omega\right) d s} P^{-} u^{0} \\
& +\int_{0}^{t} e^{A(t-s)+\int_{s}^{t} z\left(\theta_{r} \omega\right) d r} P^{-} G\left(\theta_{s} \omega, u\right) d s . \\
P^{+} u\left(t, u^{0}, \omega\right)= & e^{A(t-\tau)+\int_{\tau}^{t} z\left(\theta_{s} \omega\right) d s} P^{+} u\left(\tau, u^{0}, \omega\right) \\
& +\int_{\tau}^{t} e^{A(t-s)+\int_{s}^{t} z\left(\theta_{r} \omega\right) d r} P^{-} G\left(\theta_{s} \omega, u\right) d s .
\end{aligned}
$$

Since $u \in C_{\eta}^{+}$, we have for $t<\tau, 0<\tau$ that

$$
\begin{aligned}
& \left|e^{A(t-\tau)+\int_{\tau}^{t} z\left(\theta_{s} \omega\right) d s} P^{+} u\left(\tau, u^{0}, \omega\right)\right| \\
& \leq e^{\alpha(t-\tau)} e^{\int_{0}^{t} z\left(\theta_{s} \omega\right) d s} e^{\eta \tau}|u|_{C_{\eta}^{+}} \\
& =e^{\alpha t+\int_{0}^{t} z\left(\theta_{s} \omega\right) d s} e^{-(\alpha-\eta) \tau} \rightarrow 0 \text { as } \tau \rightarrow+\infty .
\end{aligned}
$$

Then, taking the limit $\tau \rightarrow+\infty$ in (18), we have that

$$
P^{+} u\left(t, u^{0}, \omega\right)=\int_{\infty}^{t} e^{A(t-s)+\int_{s}^{t} z\left(\theta_{r} \omega\right) d r} P^{+} G\left(\theta_{s} \omega, u(s)\right) d s .
$$

Combining (17) and (19), we have (16). The converse follows from a direct computation.

Next we prove that for any given $\xi \in H^{-}$the integral equation (16) has a unique solution in $C_{\eta}^{+}$. To see this, let $J^{s}(u, \xi)$ denote the right hand side of equality (16). It is easy to see that $J^{s}$ is well-defined from $C_{\eta}^{+} \times H^{-}$to $C_{\eta}^{+}$. For each $u, \bar{u} \in C_{\eta}^{+}$, we have that

$$
\begin{aligned}
& \left|J^{s}(u, \xi)-J^{s}(\bar{u}, \xi)\right|_{C_{\eta}^{+}} \\
& \leq \sup _{t \in[0, \infty)}\left\{e ^ { - \eta t - \int _ { 0 } ^ { t } z ( \theta _ { s } \omega ) d s } \left(\mid \int_{0}^{t} e^{A(t-s)+\int_{s}^{t} z\left(\theta_{r} \omega\right) d r} P^{-}\left(G\left(\theta_{s} \omega, u\right)\right.\right.\right. \\
& \left.-G\left(\theta_{s} \omega, \bar{u}\right)\right) d s \\
& \left.\left.+\int_{\infty}^{t} e^{A(t-s)+\int_{s}^{t} z\left(\theta_{r} \omega\right) d r} P^{+}\left(G\left(\theta_{s} \omega, u\right)-G\left(\theta_{s} \omega, \bar{u}\right)\right) d s \mid\right)\right\} \\
& \leq \sup _{t \in[0, \infty)}\left\{K \operatorname{Lip}_{u} G|u-\bar{u}|_{C_{\eta}^{+}}\left(\int_{0}^{t} e^{(\beta-\eta)(t-s)} d s+\int_{\infty}^{t} e^{(\alpha-\eta)(t-s)} d s\right)\right\} \\
& \leq K \operatorname{Lip}_{u} G\left(\frac{1}{\eta-\beta}+\frac{1}{\alpha-\eta}\right)|u-\bar{u}|_{C_{\eta}^{+} .}
\end{aligned}
$$

Obviously $J^{s}$ is Lipschitz continuous in $\xi$. By the assumption, $K \operatorname{Lip}_{u} G\left(\frac{1}{\eta-\beta}+\right.$ $\left.\frac{1}{\alpha-\eta}\right)<1$, hence $J^{s}$ is a uniform contraction with respect to the parameter $\xi$. By the uniform contraction mapping principle, we have that for each $\xi \in H^{-}$, the mapping $J^{s}(\cdot, \xi)$ has a unique fixed point $u(\cdot ; \xi, \omega) \in C_{\eta}^{+}$and $u(\cdot ; \cdot, \omega)$ is Lipschitz from $H^{-}$to $C_{\eta}^{+}$, that is, $u(\cdot ; \cdot, \omega) \in C_{\eta}^{+}$is a unique solution of the integral equation 
16). Furthermore one has for the fixed point $u$ the estimate

$$
|u(\cdot ; \xi, \omega)-u(\cdot ; \bar{\xi}, \omega)|_{C_{\eta}^{+}} \leq \frac{K}{1-K \operatorname{Lip}_{u} G\left(\frac{1}{\eta-\beta}+\frac{1}{\alpha-\eta}\right)}|\xi-\bar{\xi}| .
$$

Since $u(\cdot ; \xi, \omega)$ can be an $\omega$-wise limit of the iteration of contraction mapping $J^{s}$ starting at 0 and $J^{s}$ maps a $\mathcal{F}$-measurable function to a measurable function, $u(\cdot ; \xi, \omega)$ is $\mathcal{F}$-measurable. On the other hand, since $u(\cdot ; \xi, \omega)$ is Lipschitz continuous, by Castaing and Valadier [6], Lemma III.14, the above terms are measurable with respect to $(\xi, \omega, y)$.

Let $h^{s}(\xi, \omega)=P^{+} u(0 ; \xi, \omega)$. Then

$$
h^{s}(\xi, \omega)=\int_{\infty}^{0} e^{-A s \int_{s}^{0} z\left(\theta_{r} \omega\right) d r} P^{+} G\left(\theta_{s} \omega, u(s ; \xi, \omega)\right) d s
$$

and $h^{s}(0, \omega)=0$.

Thus, by using (21), we obtain that

$$
\left|h^{s}(\xi, \omega)-h^{s}(\bar{\xi}, \omega)\right| \leq \frac{K^{2} \operatorname{Lip}_{u} G}{(\alpha-\eta)\left(1-K \operatorname{Lip}_{u} G\left(\frac{1}{\eta-\beta}+\frac{1}{\alpha-\eta}\right)\right.}|\xi-\bar{\xi}|
$$

and $h^{s}$ is measurable. From the definition of $h^{s}(\xi, \omega)$ and the claim that $u^{0} \in M^{s}(\omega)$ if and only if there exists $u \in C_{\eta}^{+}$with $u(0)=u_{0}$ and satisfies (16) it follows that $u^{0} \in M^{s}(\omega)$ if and only if there exists $\xi \in H^{-}$such that $u^{0}=\xi+h^{s}(\xi, \omega)$, therefore,

$$
M^{s}(\omega)=\left\{\xi+h^{s}(\xi, \omega) \mid \xi \in H^{-}\right\} .
$$

In order to see that $M^{s}(\omega)$ is a random set we need to show that for any $x \in H$

$$
\omega \rightarrow \inf _{y \in H}\left\|x-\left(P^{-} y+h^{s}\left(P^{-} y, \omega\right)\right)\right\|
$$

is measurable, see Castaing and Valadier [6], Theorem III.9. Let $H_{c}$ be a countable dense set of the separable space $H$. Then the right hand side of (22) is equal to

$$
\left.\inf _{y \in H_{c}}|| x-P^{-} y+h^{s}\left(P^{-} y, \omega\right)\right) \mid
$$

which follows immediately by the continuity of $h^{s}(\cdot, \omega)$. The measurability of any expression under the infimum of (22) follows since $\omega \rightarrow h^{s}\left(P^{-} y, \omega\right)$ is measurable for any $y \in H$.

Finally, we show that $M^{s}(\omega)$ is invariant, i.e., for each $u_{0} \in M^{s}(\omega), u\left(s, u_{0}, \omega\right) \in$ $M^{s}\left(\theta_{s} \omega\right)$ for all $s \geq 0$. We first note that for each fixed $s \geq 0, u\left(t+s, u_{0}, \omega\right)$ is a solution of

$$
\frac{d u}{d t}=A u+z\left(\theta_{t}\left(\theta_{s} \omega\right)\right) u+G\left(\theta_{t}\left(\theta_{s} \omega\right), u\right), \quad u(0)=u\left(s, u_{0}, \omega\right) .
$$

Thus, $u\left(t, u\left(s, u_{0}, \omega\right), \theta_{s} \omega\right)=u\left(t+s, u_{0}, \omega\right)$.

Since $u\left(\cdot, u_{0}, \omega\right) \in C_{\eta}^{+}, u\left(t, u\left(s, u_{0}, \omega\right), \theta_{s} \omega\right) \in C_{\eta}^{+}$. Therefore, $u\left(s, u_{0}, \omega\right) \in M^{s}\left(\theta_{s} \omega\right)$ This completes the proof.

Theorem 3.2. $\tilde{M}^{s}(\omega)=T^{-1}\left(\omega, M^{s}(\omega)\right)$ is a Lipschiz stable manifold of the stochastic partial differential equation (5). 
Proof. Let $u(t, \omega, x)$ denote the solution of (8) and $\tilde{u}(t, \omega, x)$ denote the solution of (5). From Lemma 2.2 we have

$$
\begin{aligned}
& \tilde{u}\left(t, \omega, \tilde{M}^{s}(\omega)\right)=T^{-1}\left(\theta_{t} \omega, u\left(t, \omega, T\left(\omega, \tilde{M}^{s}(\omega)\right)\right)\right) \\
& \quad=T^{-1}\left(\theta_{t} \omega, u\left(t, \omega, M^{s}(\omega)\right)\right) \subset T^{-1}\left(\theta_{t} \omega, M^{s}\left(\theta_{t} \omega\right)\right)=\tilde{M}^{s}\left(\theta_{t} \omega\right) .
\end{aligned}
$$

Hence, $\tilde{M}^{s}(\omega)$ is an invariant set. We also notice that

$$
\begin{aligned}
& \tilde{M}^{s}(\omega) \\
& =T^{-1}\left(\omega, M^{s}(\omega)\right) \\
& =\left\{u_{0}=T^{-1}\left(\omega, \xi+h^{s}(\xi, \omega) \mid \xi \in H^{-}\right\}\right. \\
& =\left\{u_{0}=e^{z(\omega)}\left(\xi+h^{s}(\xi, \omega)\right) \mid \xi \in H^{-}\right\} \\
& =\left\{u_{0}=\left(\xi+h^{s}\left(e^{-z(\omega)} \xi, \omega\right)\right) \mid \xi \in H^{-}\right\}
\end{aligned}
$$

which implies that $\tilde{M}^{s}(\omega)$ is a Lipschitz stable manifold given by the graph of a Lipschitz continuous function $\tilde{h}^{s}(\xi, \omega)=h^{s}\left(e^{-z(\omega)} \xi, \omega\right)$ over the space $H^{-}$.

\section{Smoothness of Stable Manifolds}

In this section, we prove that for each $\omega, M^{s}(\omega)$ is a $C^{k}$ smooth manifold. We have

Theorem 4.1. Assume that $G$ is $C^{k}$ in $u$. If $\beta<k \eta<\alpha$ and

$$
K \operatorname{Lip}_{u} G\left(\frac{1}{i \eta-\beta}+\frac{1}{\alpha-i \eta}\right)<1 \quad \text { for all } 1 \leq i \leq k,
$$

then $M^{s}(\omega)$ is a $C^{k}$ invariant stable manifold for the random partial differential equation (15), i.e., $h(\xi, \omega)$ is $C^{k}$ in $\xi$.

Proof. We prove this theorem by induction. First, we consider $k=1$. Since

$$
K \operatorname{Lip}_{u} G\left(\frac{1}{\eta-\beta}+\frac{1}{\alpha-\eta}\right)<1
$$

there exists a small number $\delta>0$ such that $\beta<\eta-2 \delta$ and

$$
K \operatorname{Lip}_{u} G\left(\frac{1}{(\eta-\gamma)-\beta}+\frac{1}{\alpha-(\eta-\gamma)}\right)<1 \quad \text { for all } 0 \leq \gamma \leq 2 \delta
$$

Thus, $J^{s}(\cdot, \xi, \omega)$ defined in the proof of Theorem 3.1 is a uniform contraction in $C_{\eta-\gamma}^{+} \subset C_{\eta}^{+}$for any $0 \leq \gamma \leq 2 \delta$. Therefore, $u(\cdot ; \xi, \omega) \in C_{\eta-\gamma}^{+}$. For $\xi_{0} \in H^{-}$, we set

$$
S=e^{A t+\int_{0}^{t} z\left(\theta_{s} \omega\right) d s},
$$

and

$$
\begin{aligned}
T v & =\int_{0}^{t} e^{A(t-s)+\int_{s}^{t} z\left(\theta_{r} \omega\right) d r} P^{-} D_{u} G\left(\theta_{s} \omega, u\left(s ; \xi_{0}, \omega\right)\right) v d s \\
& +\int_{\infty}^{t} e^{A(t-s)+\int_{s}^{t} z\left(\theta_{r} \omega\right) d r} P^{+} D_{u} G\left(\theta_{s} \omega, u\left(s ; \xi_{0}, \omega\right)\right) v d s
\end{aligned}
$$

for $v \in C_{\eta-\delta}^{+}$. From the assumption, we have that $S$ is a bounded linear operator from $H^{-}$to $C_{\eta-\delta}^{+}$. Using the same arguments as we proved that $J^{s}$ is a contraction, we have that $T$ is a bounded linear operator from $C_{\eta-\delta}^{+}$to itself and

$$
\|T\| \leq K \operatorname{Lip}_{u} G\left(\frac{1}{(\eta-\delta)-\beta}+\frac{1}{\alpha-(\eta-\delta)}\right)<1,
$$


which implies that $I d-T$ is invertible in $C_{\eta-\delta}^{+}$. For $\xi, \xi_{0} \in H^{-}$, we set

$$
\begin{array}{r}
I=\int_{0}^{t} e^{A(t-s)+\int_{s}^{t} z\left(\theta_{r} \omega\right) d r} P^{-}\left[G\left(\theta_{s} \omega, u(s ; \xi, \omega)\right)-G\left(\theta_{s} \omega, u\left(s ; \xi_{0}, \omega\right)\right)\right. \\
\left.-D_{u} G\left(\theta_{s} \omega, u\left(s ; \xi_{0}, \omega\right)\right)\left(u(s ; \xi, \omega)-u\left(s ; \xi_{0}, \omega\right)\right)\right] d s \\
+\int_{\infty}^{t} e^{A(t-s)+\int_{s}^{t} z\left(\theta_{r} \omega\right) d r} P^{+}\left[G\left(\theta_{s} \omega, u(s ; \xi, \omega)\right)-G\left(\theta_{s} \omega, u\left(s ; \xi_{0}, \omega\right)\right)\right. \\
\left.-D_{u} G\left(\theta_{s} \omega, u\left(s ; \xi_{0}, \omega\right)\right)\left(u(s ; \xi, \omega)-u\left(s ; \xi_{0}, \omega\right)\right)\right] d s .
\end{array}
$$

We claim that $|I|_{C_{\eta-\delta}^{+}}=o\left(\left|\xi-\xi_{0}\right|\right)$ as $\xi \rightarrow \xi_{0}$. Using this claim, we obtain

$$
\begin{aligned}
& u(\cdot ; \xi, \omega)-u\left(\cdot ; \xi_{0}, \omega\right)-T\left(u(\cdot ; \xi, \omega)-u\left(\cdot ; \xi_{0}, \omega\right)\right) \\
& =S\left(\xi-\xi_{0}\right)+I \\
& =S\left(\xi-\xi_{0}\right)+o\left(\left|\xi-\xi_{0}\right|\right), \text { as } \xi \rightarrow \xi_{0} .
\end{aligned}
$$

which yields

$$
u(\cdot ; \xi, \omega)-u\left(\cdot ; \xi_{0}, \omega\right)=(I d-T)^{-1} S\left(\xi-\xi_{0}\right)+o\left(\left|\xi-\xi_{0}\right|\right) .
$$

Hence, $u(\cdot ; \xi, \omega)$ is differentiable in $\xi$ and its derivative satisfies $D_{\xi} u(t ; \xi, \omega) \in$ $L\left(H^{-}, C_{\eta-\delta}^{+}\right)$, where $L\left(H^{-}, C_{\eta-\delta}^{+}\right)$is the usual space of bounded linear operators and

$$
\begin{aligned}
D_{\xi} u(t ; \xi, \omega)= & e^{A t+\int_{0}^{t} z\left(\theta_{s} \omega\right) d s} P^{-} . \\
& +\int_{0}^{t} e^{A(t-s) \int_{s}^{t}+z\left(\theta_{r} \omega\right) d r} P^{-} D_{u} G\left(\theta_{s} \omega, u(s ; \xi, \omega)\right) D_{\xi} u(s ; \xi, \omega) d s \\
& +\int_{\infty}^{t} e^{A(t-s)+\int_{s}^{t} z\left(\theta_{r} \omega\right) d r} P^{+} D_{u} G\left(\theta_{s} \omega, u(s ; \xi, \omega)\right) D_{\xi} u(s ; \xi, \omega) d s
\end{aligned}
$$

Now we prove that $|I|_{C_{\eta-\delta}^{+}}=o\left(\left|\xi-\xi_{0}\right|\right)$ as $\xi \rightarrow \xi_{0}$. Let $N$ be a large positive number to be chosen later and let

$$
\begin{aligned}
I_{1}= & e^{-(\eta-\delta) t-\int_{0}^{t} z\left(\theta_{s} \omega\right) d s}\left\{\mid \int_{N}^{t} e^{A(t-s)+\int_{s}^{t} z\left(\theta_{r} \omega\right) d r} P^{-}\left[G\left(\theta_{s} \omega, u(s ; \xi, \omega)\right)\right.\right. \\
& \left.\left.-G\left(\theta_{s} \omega, u\left(s ; \xi_{0}, \omega\right)\right)-D_{u} G\left(\theta_{s} \omega, u\left(s ; \xi_{0}, \omega\right)\right)\left(u(s ; \xi, \omega)-u\left(s ; \xi_{0}, \omega\right)\right)\right] d s \mid\right\}
\end{aligned}
$$

for $t \geq N$ and $I_{1}=0$ for $t<N$;

$$
\begin{aligned}
I_{2}= & e^{-(\eta-\delta) t-\int_{0}^{t} z\left(\theta_{s} \omega\right) d s}\left\{\mid \int_{0}^{N} e^{A(t-s)+\int_{s}^{t} z\left(\theta_{r} \omega\right) d r} P^{-}\left[G\left(\theta_{s} \omega, u(s ; \xi, \omega)\right)\right.\right. \\
& \left.\left.-G\left(\theta_{s} \omega, u\left(s ; \xi_{0}, \omega\right)\right)-D_{u} G\left(\theta_{s} \omega, u\left(s ; \xi_{0}, \omega\right)\right)\left(u(s ; \xi, \omega)-u\left(s ; \xi_{0}, \omega\right)\right)\right] d s \mid\right\} .
\end{aligned}
$$


Let $\bar{N}$ be a large positive number to be chosen later. For $0 \leq t \leq \bar{N}$, we set

$$
\begin{aligned}
I_{3}= & e^{-(\eta-\delta) t-\int_{t}^{0} z\left(\theta_{s} \omega\right) d s}\left\{\mid \int_{\bar{N}}^{t} e^{A(t-s)+\int_{s}^{t} z\left(\theta_{r} \omega\right) d r} P^{+}\left[G\left(\theta_{s} \omega, u(s ; \xi, \omega)\right)\right.\right. \\
& \left.\left.-G\left(\theta_{s} \omega, u\left(s ; \xi_{0}, \omega\right)\right)-D_{u} G\left(\theta_{s} \omega, u\left(s ; \xi_{0}, \omega\right)\right)\left(u(s ; \xi, \omega)-u\left(s ; \xi_{0}, \omega\right)\right)\right] d s \mid\right\} ; \\
I_{4}= & e^{-(\eta-\delta) t-\int_{t}^{0} z\left(\theta_{s} \omega\right) d s}\left\{\mid \int_{\infty}^{\bar{N}} e^{A(t-s)+\int_{s}^{t} z\left(\theta_{r} \omega\right) d r} P^{+}\left[G\left(\theta_{s} \omega, u(s ; \xi, \omega)\right)\right.\right. \\
& \left.\left.-G\left(\theta_{s} \omega, u\left(s ; \xi_{0}, \omega\right)\right)-D_{u} G\left(\theta_{s} \omega, u\left(s ; \xi_{0}, \omega\right)\right)\left(u(s ; \xi, \omega)-u\left(s ; \xi_{0}, \omega\right)\right)\right] d s \mid\right\} .
\end{aligned}
$$

For $t \geq \bar{N}$, we set

$$
\begin{aligned}
I_{5}= & e^{-(\eta-\delta) t-\int_{t}^{0} z\left(\theta_{s} \omega\right) d s}\left\{\mid \int_{\infty}^{t} e^{A(t-s)+\int_{s}^{t} z\left(\theta_{r} \omega\right) d r} P^{+}\left[G\left(\theta_{s} \omega, u(s ; \xi, \omega)\right)\right.\right. \\
& \left.\left.-G\left(\theta_{s} \omega, u\left(s ; \xi_{0}, \omega\right)\right)-D_{u} G\left(\theta_{s} \omega, u\left(s ; \xi_{0}, \omega\right)\right)\left(u(s ; \xi, \omega)-u\left(s ; \xi_{0}, \omega\right)\right)\right] d s \mid\right\} .
\end{aligned}
$$

It is sufficient to show that for any $\epsilon>0$ there is a $\sigma>0$ such that if $\left|\xi-\xi_{0}\right| \leq \sigma$, then $|I|_{C_{\eta-\delta}^{+}} \leq \epsilon\left|\xi-\xi_{0}\right|$. Note that

$$
|I|_{C_{\eta-\delta}^{+}} \leq \sup _{t \geq 0} I_{1}+\sup _{t \geq 0} I_{2}+\sup _{0 \leq t \leq \bar{N}} I_{3}+\sup _{0 \leq t \leq \bar{N}} I_{4}+\sup _{t \geq \bar{N}} I_{5}
$$

A computation similar to (21) implies that

$$
\begin{aligned}
I_{1} & \leq 2 K \operatorname{Lip}_{u} G \int_{N}^{t} e^{(\beta-(\eta-\delta))(t-s)} e^{-\delta s}\left|u(\cdot ; \xi, \omega)-u\left(\cdot ; \xi_{0}, \omega\right)\right|_{C_{\eta-2 \delta}^{+}} d s \\
& \leq \frac{2 K^{2} \operatorname{Lip}_{u} G e^{-\delta N}}{(\eta-\delta-\beta)\left(1-K \operatorname{Lip}_{u} G\left(\frac{1}{\eta-2 \delta-\beta}+\frac{1}{\alpha-(\eta-2 \delta)}\right)\right)}\left|\xi-\xi_{0}\right| .
\end{aligned}
$$

Choose $N$ so large that

$$
\frac{2 K^{2} \operatorname{Lip}_{u} G e^{-\delta N}}{(\eta-\delta-\beta)\left(1-K \operatorname{Lip}_{u} G\left(\frac{1}{\eta-2 \delta-\beta}+\frac{1}{\alpha-(\eta-2 \delta)}\right)\right)} \leq \frac{1}{4} \epsilon .
$$

Hence for such $N$ we have that

$$
\sup _{t \geq 0} I_{1} \leq \frac{1}{4} \epsilon\left|\xi-\xi_{0}\right|_{X} .
$$


Fixing such $N$, for $I_{2}$ we have that

$$
\begin{aligned}
I_{2} & \leq K \int_{0}^{N} e^{(\beta-(\eta-\delta))(t-s)}\left\{\int _ { 0 } ^ { 1 } \left[\mid D_{u} G\left(\theta_{s} \omega, \tau u(s ; \xi, \omega)+(1-\tau) u\left(s ; \xi_{0}, \omega\right)\right)\right.\right. \\
& \left.\left.-D_{u} G\left(\theta_{s} \omega, u\left(s ; \xi_{0}, \omega\right)\right) \mid\right] d \tau\right\}\left|u(\cdot ; \xi, \omega)-u\left(\cdot ; \xi_{0}, \omega\right)\right|_{C_{\eta-\delta}^{+}} d s \\
& \leq \frac{K^{2}\left|\xi-\xi_{0}\right|}{1-K \operatorname{Lip}_{u} G\left(\frac{1}{\eta-\delta-\beta}+\frac{1}{\alpha-(\eta-\delta)}\right)} \\
& \int_{0}^{N} e^{-(\beta-(\eta-\delta)) s}\left\{\int _ { 0 } ^ { 1 } \left[\mid D_{u} G\left(\theta_{s} \omega, \tau u(s ; \xi, \omega)+(1-\tau) u\left(s ; \xi_{0}, \omega\right)\right)\right.\right. \\
& \left.\left.-D_{u} G\left(\theta_{s} \omega, u\left(s ; \xi_{0}, \omega\right)\right) \mid\right] d \tau\right\} d s .
\end{aligned}
$$

The last integral is on the compact interval $[0, N]$. Thus, from the continuity of the integrand $(s, \xi)$, we have that there is a $\sigma_{1}>0$ such that if $\left|\xi-\xi_{0}\right| \leq \sigma_{1}$, then

$$
\sup _{t \geq 0} I_{2} \leq \frac{1}{4} \epsilon\left|\xi-\xi_{0}\right|
$$

Therefore, if $\left|\xi-\xi_{0}\right| \leq \sigma_{1}$, then

$$
\sup _{t \geq 0} I_{1}+\sup _{t \geq 0} I_{2} \leq \frac{1}{2} \epsilon\left|\xi-\xi_{0}\right|
$$

Similarly, by choosing $\bar{N}$ to be sufficiently large, we have

$$
\sup _{0 \leq t \leq \bar{N}} I_{4}+\sup _{t \geq \bar{N}} I_{5} \leq \frac{1}{4} \epsilon\left|\xi-\xi_{0}\right|
$$

and for fixed such $\bar{N}$, there exists $\sigma_{2}>0$ such that if $\left|\xi-\xi_{0}\right| \leq \sigma_{2}$, then

$$
\sup _{0 \leq t \leq \bar{N}} I_{3} \leq \frac{1}{4} \epsilon\left|\xi_{1}-\xi_{2}\right|
$$

Taking $\sigma=\min \left\{\sigma_{1}, \sigma_{2}\right\}$, we have that if $\left|\xi-\xi_{0}\right| \leq \sigma$, then

$$
|I|_{C_{\eta-\delta}^{+}} \leq \epsilon\left|\xi-\xi_{0}\right|
$$

Therefore $|I|_{C_{\eta-\delta}^{+}}=o\left(\left|\xi-\xi_{0}\right|\right)$ as $\xi \rightarrow \xi_{0}$. We now prove that $D_{\xi} u(\cdot ; \cdot, \omega)$ is continuous from $H^{-}$to $C_{\eta}^{+}$). For $\xi, \xi_{0} \in H^{-}$, using (25), we have 


$$
\begin{gathered}
D_{\xi} u(t ; \xi, \omega)-D_{\xi} u\left(t ; \xi_{0}, \omega\right) \\
=\int_{0}^{t} e^{A(t-s)+\int_{s}^{t} z\left(\theta_{r} \omega\right) d r} P^{-}\left(D_{u} G\left(\theta_{s} \omega, u(s ; \xi, \omega)\right) D_{\xi} u(s ; \xi, \omega)\right. \\
\left.-D_{u} G\left(\theta_{s} \omega, u\left(s ; \xi_{0}, \omega\right)\right) D_{\xi} u\left(s ; \xi_{0}, \omega\right)\right) d s \\
+\int_{\infty}^{t} e^{A(t-s)+\int_{s}^{t} z\left(\theta_{r} \omega\right) d r} P^{+}\left(D_{u} G\left(\theta_{s} \omega, u(s ; \xi, \omega)\right) D_{\xi} u(s ; \xi, \omega)\right. \\
\left.-D_{u} G\left(\theta_{s} \omega, u\left(s ; \xi_{0}, \omega\right)\right) D_{\xi} u\left(s ; \xi_{0}, \omega\right)\right) d s \\
=\int_{0}^{t} e^{A(t-s)+\int_{s}^{t} z\left(\theta_{r} \omega\right) d r} P^{-}\left(D_{u} G\left(\theta_{s} \omega, u(s ; \xi, \omega)\right)\right. \\
\left.\quad\left(D_{\xi} u(s ; \xi, \omega)-D_{\xi} u\left(s ; \xi_{0}, \omega\right)\right)\right) d s \\
+\int_{\infty}^{t} e^{A(t-s)+\int_{s}^{t} z\left(\theta_{r} \omega\right) d r} P^{+}\left(D_{u} G\left(\theta_{s} \omega, u(s ; \xi, \omega)\right)\right. \\
\left.\left(D_{\xi} u(s ; \xi, \omega)-D_{\xi} u\left(s ; \xi_{0}, \omega\right)\right)\right) d s+\bar{I},
\end{gathered}
$$

where

$$
\begin{aligned}
\bar{I}=\int_{0}^{t} e^{A(t-s)+\int_{s}^{t} z\left(\theta_{r} \omega\right) d r} P^{-} & \left(D_{u} G\left(\theta_{s} \omega, u(s ; \xi, \omega)\right)\right. \\
& -D_{u} G\left(\theta_{s} \omega, u\left(s ; \xi_{0}, \omega\right)\right) D_{\xi} u\left(s ; \xi_{0}, \omega\right) d s \\
+\int_{\infty}^{t} e^{A(t-s)+\int_{s}^{t} z\left(\theta_{r} \omega\right) d r} & P^{+}\left(D_{u} G\left(\theta_{s} \omega, u(s ; \xi, \omega)\right)\right. \\
& -D_{u} G\left(\theta_{s} \omega, u\left(s ; \xi_{0}, \omega\right)\right) D_{\xi} u\left(s ; \xi_{0}, \omega\right) d s .
\end{aligned}
$$

Then, estimating $\left|D_{\xi} u(\cdot ; \xi, \omega)-D_{\xi} u\left(\cdot ; \xi_{0}, \omega\right)\right|_{L\left(H^{-}, C_{\eta}^{+}\right)}$, we have

$$
\begin{aligned}
& \left|D_{\xi} u(\cdot ; \xi, \omega)-D_{\xi} u\left(\cdot ; \xi_{0}, \omega\right)\right|_{L\left(H^{-}, C_{\eta}^{+}\right)} \\
& \leq \frac{|\bar{I}|_{L\left(H^{-}, C_{\eta}^{+}\right)}}{1-K \operatorname{Lip}_{u} G\left(\frac{1}{\eta-\beta}+\frac{1}{\alpha-\eta}\right)} .
\end{aligned}
$$

Using the same argument we used for the last claim, we obtain that $|\bar{I}|_{L\left(H^{-}, C_{\eta}^{+}\right)}=$ $o(1)$ as $\xi \rightarrow \xi_{0}$. Hence $D_{\xi} u(\cdot ; \cdot, \omega)$ is continuous from $H^{-}$to $L\left(H^{-}, C_{\eta}^{+}\right)$. Therefore, $u(\cdot ; \cdot, \omega)$ is $C^{1}$ from $H^{-}$to $C_{\eta}^{+}$. Now we show that $u$ is $C^{k}$ from $H^{-}$to $C_{k \eta}^{+}$by induction for $k \geq 2$. By the induction assumption, we know that $u$ is $C^{k-1}$ from $H^{-}$to $C_{(k-1) \eta}^{+}$and the and $(k-1)$-derivative $D_{\xi}^{k-1} u(t ; \xi, \omega)$ satisfies the following 
equation

$$
\begin{aligned}
D_{\xi}^{k-1} u= & \int_{0}^{t} e^{A(t-s) \int_{s}^{t}+z\left(\theta_{r} \omega\right) d r} P^{-}\left(D_{u} G\left(\theta_{s} \omega, u\right) D_{\xi}^{k-1} u d s\right. \\
& +\int_{\infty}^{t} e^{A(t-s)+\int_{s}^{t} z\left(\theta_{r} \omega\right) d r} P^{+} D_{u} G\left(\theta_{s} \omega, u\right) D_{\xi}^{k-1} u d s \\
& \int_{0}^{t} e^{A(t-s) \int_{s}^{t}+z\left(\theta_{r} \omega\right) d r} P^{-} R_{k-1}(s, \xi, \omega) d s \\
& +\int_{\infty}^{t} e^{A(t-s)+\int_{s}^{t} z\left(\theta_{r} \omega\right) d r} P^{+} R_{k-1}(s, \xi, \omega) d s
\end{aligned}
$$

where

$$
R_{k-1}(s, \xi, \omega)=\sum_{i=0}^{k-3}\left(\begin{array}{c}
k-2 \\
i
\end{array}\right) D_{\xi}^{k-2-i}\left(D_{u} G\left(\theta_{s} \omega, u(s ; \xi, \omega)\right)\right) D_{\xi}^{i+1} u(s ; \xi, \omega) .
$$

We note that $D_{\xi}^{i} u \in C_{i \eta}^{+}$for $i=1, \cdots, k-1$ from the induction hypothesis. Thus, using the fact that $G$ is $C^{k}$, we can verify that $R_{k-1}(\cdot, \xi, \omega) \in L^{k-1}\left(H^{-}, C_{(k-1) \eta}^{+}\right)$ and is $C^{1}$ in $\xi$, where $L^{k-1}\left(H^{-}, C_{(k-1) \eta}^{+}\right)$is the usual space of bounded $k-1$ linear forms. In order to insure that the above integrals are well-defined one has to require that $\beta<(k-1) \eta<\alpha$. This is the reason why we need the gap condition. The fact that $t \rightarrow z\left(\theta_{t} \omega\right)$ has a sublinear growth rate is also used in these analysis. Note that from the assumption $\beta<k \eta<\alpha$ and

$$
K \operatorname{Lip}_{u} G\left(\frac{1}{i \eta-\beta}+\frac{1}{\alpha-i \eta}\right)<1 \quad \text { for all } 1 \leq i \leq k .
$$

Using this fact and the same argument which we used in the case $k=1$, we can show that $D_{\xi}^{k-1} u(\cdot ; \cdot, \omega)$ is $C^{1}$ from $X$ to $L^{k}\left(H^{-}, C_{k \eta}^{+}\right)$. This completes the proof.

Theorem 4.2. Assume that $F(u)$ is $C^{k}$ smooth. If $\beta<k \eta<\alpha$ and

$$
K \operatorname{Lip}_{u} G\left(\frac{1}{i \eta-\beta}+\frac{1}{\alpha-i \eta}\right)<1 \quad \text { for all } 1 \leq i \leq k,
$$

then $\tilde{M}^{s}(\omega)=T^{-1}\left(\omega, M^{s}(\omega)\right)$ is a $C^{k}$ invariant stable manifold for the stochastic partial differential equation (5).

Proof. Since

$$
\tilde{M}^{s}(\omega)=\left\{\xi+\tilde{h}^{s}(\xi, \omega) \mid \xi \in H^{-}\right\},
$$

$\tilde{h}^{s}(\xi, \omega)=e^{z} h^{s}\left(e^{-z(\omega)} \xi, \omega\right)$, and $h^{s}(\xi, \omega)$ is $C^{k}$ in $\xi, \tilde{h}^{s}(\xi, \omega)$ is $C^{k}$ in $\xi$.

\section{Smooth Unstable Manifolds}

All results obtained in Section 3 and Section 4 also hold for unstable manifolds.

Theorem 5.1. If

$$
K \operatorname{Lip}_{u} G\left(\frac{1}{\eta-\beta}+\frac{1}{\alpha-\eta}\right)<1
$$

then there exists a Lipschitz unstable manifold for the random partial differential equation [15), which is given by

$$
M^{u}(\omega)=\left\{\xi+h^{u}(\xi, \omega) \mid \xi \in H^{+}\right\}
$$


where $h^{u}: H^{+} \rightarrow H^{-}$is a Lipschitz continuous mapping and satisfies $h^{u}(0)=0$. Moreover, $\tilde{M}^{u}(\omega)=T^{-1}\left(\omega, M^{u}(\omega)\right)$ is a Lipschiz stable manifold of the stochastic partial differential equation (5).

Theorem 5.2. Assume that the nonlinear term $F$ and thus $G$ is $C^{k}$ in $u$. If $\beta<k \eta<\alpha$ and

$$
K \operatorname{Lip}_{u} G\left(\frac{1}{i \eta-\beta}+\frac{1}{\alpha-i \eta}\right)<1 \quad \text { for all } 1 \leq i \leq k,
$$

then $M^{u}(\omega)$ is a $C^{k}$ unstable manifold for the random partial differential equation [15), i.e., $h^{u}(\xi, \omega)$ is $C^{k}$ in $\xi$. Moreover, $\tilde{M}^{u}(\omega)=T^{-1}\left(\omega, M^{u}(\omega)\right)$ is a $C^{k}$ unstable manifold for the stochastic partial differential equation (5).

Generally, a few modifications are needed to adapt the proofs presented in Section 3 and Section 4 to the case of unstable manifold. The most significant differences are the integral equation (16) and the associated function space. We shall outline the proofs and leave the details to the interested reader.

Corresponding to space $C_{\eta}^{+}$, we define the Banach Space for each $\beta<\eta<\alpha$

$$
C_{\eta}^{-}=\left\{\phi:(-\infty, 0] \rightarrow H \mid \phi \text { is continuous and } \sup _{t \leq 0} e^{-\eta t-\int_{0}^{t} z\left(\theta_{\tau} \omega\right) d \tau}|\phi(t)|<\infty\right\}
$$

with the norm

$$
|\phi|_{C_{\eta}^{-}}=\sup _{t \leq 0} e^{-\eta t-\int_{0}^{t} z\left(\theta_{\tau} \omega\right) d \tau}|\phi(t)| .
$$

Let

$$
M^{u}(\omega)=\left\{u_{0} \in H \mid u\left(\cdot, \omega, u_{0}\right) \in C_{\eta}^{-}\right\}
$$

This is the set of all initial datum through which solutions decay as $e^{\eta t+\int_{0}^{t} z\left(\theta_{\tau} \omega\right) d \tau}$ as $t \rightarrow-\infty$.

Clearly, $M^{u}(\omega)$ is invariant. In order to show that $M^{u}(\omega)$ is given by the graph of a $C^{k}$ (or Lipschitz) function, one needs to prove that $u^{0} \in M^{u}(\omega)$ if and only if there exists a function $u(\cdot) \in C_{\eta}^{-}$with $u(0)=u^{0}$ and satisfies

$$
\begin{aligned}
u(t)= & e^{A t+\int_{0}^{t} z\left(\theta_{s} \omega\right) d s} \xi+\int_{0}^{t} e^{A(t-s)+\int_{s}^{t} z\left(\theta_{r} \omega\right) d r} P^{+} G\left(\theta_{s} \omega, u(s)\right) d s \\
& +\int_{-\infty}^{t} e^{A(t-s)+\int_{s}^{t} z\left(\theta_{r} \omega\right) d r} P^{-} G\left(\theta_{s} \omega, u(s)\right) d s .
\end{aligned}
$$

where $\xi=P^{+} u^{0}$.

The next step is to show that for any given $\xi \in H^{+}$the integral equation (27) has a unique solution in $C_{\eta}^{-}$. To see this, letting $J^{u}(u, \xi)$ denote the right hand side of integral equation (27), one may show that $J^{u}$ is a unform contraction. Hence, by the uniform contraction mapping principle, we have that for each $\xi \in H^{+}$, the mapping $J^{u}(\cdot, \xi)$ has a unique fixed point $u(\cdot ; \xi, \omega) \in C_{\eta}^{-}$and $u(\cdot ; \cdot, \omega)$ is Lipschitz from $H^{+}$to $C_{\eta}^{-}$. Thus, $u(\cdot ; \cdot, \omega) \in C_{\eta}^{-}$is a solution of integral equation (27). 
Let $h^{u}(\xi, \omega)=P^{-} u(0 ; \xi, \omega)$. Then

$$
h^{u}(\xi, \omega)=\int_{-\infty}^{0} e^{-A s \int_{s}^{0}+z\left(\theta_{r} \omega\right) d r} P^{+} G\left(\theta_{s} \omega, u(s ; \xi, \omega)\right) d s
$$

and $h^{u}(0, \omega)=0$ if $F(0)=0$ or $G(\omega, 0)=0$.

Therefore,

$$
M^{u}(\omega)=\left\{\xi+h^{u}(\xi, \omega) \mid \xi \in H^{+}\right\}
$$

In the same fashion as the case for the smoothness of stable manifold, one may show that $h^{u}$ is $C^{k}$ when the assumptions in Theorem [5.2 hold.

\section{REFERENCES}

[1] L. Arnold. Random Dynamical Systems. Springer, New York, 1998.

[2] A. B. Babin and M. I. Vishik. Attractors of Evolution Equations. North-Holland, Amsterdam, London, New York, Tokyo, 1992.

[3] P. Bates, K. Lu, and C. Zeng. Existence and Persistence of Invariant Manifolds for Semiflows in Banach Space, volume 135 of Memoirs of the AMS. 1998.

[4] T. Caraballo, J. Duan, K. Lu, and B. Schmalfuß. Local manifolds for stochastic partial differential equations. In preparation.

[5] T. Caraballo P. Kloeden and B. Schmalfuß. Exponentially stable stationary solutions for stochastic evolution equations and their perturbation. 2003. Manuscript.

[6] C. Castaing and M. Valadier. Convex Analysis and Measurable Multifunctions. LNM 580. Springer-Verlag, Berlin-Heidelberg-New York, 1977.

[7] S-N. Chow, K. Lu, and X-B. Lin. Smooth foliations for flows in Banach space. Journal of Differential Equations, 94:266-291, 1991.

[8] G. Da Prato and J. Zabczyk. Stochastic Equations in Infinite Dimensions. University Press, Cambridge, 1992.

[9] G. Da Prato and A. Debussche. Construction of stochastic inertial manifolds using backward integration. Stochastics Stochastics Rep., 59(3-4):305-324, 1996.

[10] J. Duan, K. Lu, and B. Schmalfuß. Invariant manifolds for stochastic partial differential equations. Ann. Prob., in press, 2003.

[11] J. Hadamard. Sur l'iteration et les solutions asymptotiques des equations differentielles. Bull. Soc. Math. France, 29:224-228, 1901.

[12] D. Henry. Geometric theory of semilinear parabolic equations, volume 840 of Lecture Notes in Mathematics. Springer-Verlag, New York, 1981.

[13] A. M. Liapunov. Problème géneral de la stabilité du mouvement, volume 17 of Annals Math. Studies. Princeton, N.J, 1947.

[14] H. Kunita. Stochastic Flows and Stochastic Differential Equations. Cambridge University Press, Cambridge, 1990.

[15] S.-E. A. Mohammed and M. K. R. Scheutzow. The stable manifold theorem for stochastic differential equations. The Annals of Probability, 27(2):615-652, 1999.

[16] O. Perron. Über Stabilität und asymptotisches Verhalten der Integrale von Differentialgleichungssystemen. Math. Z., 29:129-160, 1928.

[17] D. Ruelle. Characteristic exponents and invariant manifolds in Hilbert spaces. Ann. of Math., 115:243-290, 1982.

[18] B. Schmalfuß. The random attractor of the stochastic Lorenz system. ZAMP, 48:951-975, 1997.

[19] B. Schmalfuß. A random fixed point theorem and the random graph transformation. Journal of Mathematical Analysis and Applications, 225(1):91-113, 1998.

[20] B. Schmalfuß. Attractors for the non-autonomous dynamical systems. In K. Gröger, B. Fiedler and J. Sprekels, editors, Proceedings EQUADIFF99, pages 684-690. World Scientific, 2000.

[21] T. Caraballo, J. Langa and J. C. Robinson. A stochastic pitchfork bifurcation in a reactiondiffusion equation. Proc. R. Soc. Lond. A, 457:2041-2061, 2001.

[22] T. Wanner. Linearization of random dynamical systems. In C. Jones, U. Kirchgraber and H. O. Walther, editors, Dynamics Reported, Vol. 4, 203-269, Springer-Verlag, New York, 1995. 
(J. Duan) Department of Applied Mathematics, Illinois Institute of Technology, Chicago, IL 60616, USA, and, Department of Mathematics, The University of Science and TechNOLOGy of China, Hefei 230026, China

E-mail address, J. Duan: duan@iit.edu

(K. Lu) Department of Mathematics, Brigham Young University, Provo, Utah 84602, USA, and, Department of Mathematics, Michigan State University, East Lansing, MichiGAN 48824, USA

E-mail address, K. Lu: klu@math.byu.edu or klu@math.msu.edu

(B. Schmalfuß) Department of Sciences, University of Applied Sciences, Geusaer Strasse, 06217 Merseburg, Germany

E-mail address, B. Schmalfuß: bjoern.schmalfuss@in.fh-merseburg.de 